\title{
Theory of coherent transition radiation generated by ellipsoidal electron bunches
}

\author{
W. P. E. M. Op 't Root, P. W. Smorenburg, T. van Oudheusden, M. J. van der Wiel, and O. J. Luiten \\ Eindhoven University of Technology, Center for Plasma Physics and Radiation Technology, P.O. Box 513, 5600 MB Eindhoven, \\ The Netherlands
}

(Received 19 August 2006; published 8 January 2007)

\begin{abstract}
We present the theory of coherent transition radiation (CTR) generated by ellipsoidal electron bunches. We calculate analytical expressions for the electric field spectrum, the power spectrum, and the temporal electric field of CTR, generated by cylindrically symmetric ellipsoidal electron bunches with hard and soft edges. This theory is relevant for diagnostics of ellipsoidal electron bunches. Realization of such bunches would solve the problem of space-charge induced emittance degradation.
\end{abstract}

DOI: 10.1103/PhysRevSTAB.10.012802

PACS numbers: $41.60 .-\mathrm{m}$

\section{INTRODUCTION}

For a long time it has been realized that uniformly filled ellipsoidal electron bunches, also known as "waterbag" bunches, are the ideal particle distributions for controlled, high-brightness charged particle acceleration. Because of their linear internal fields they do not suffer from brightness degradation caused by space-charge forces $[1,2]$. In spite of this they were never considered as a realistic option, because there was no method to create them. In 1997, Serafini was the first to suggest that waterbag bunches may be created by using high-gradient radiofrequency photoguns, operated in the space-charge blowout regime [3]. In 2004, Luiten et al. presented a practical recipe which results in an almost ideal hard-edged ellipsoidal electron bunch [4-6]. The deviation from the ideal situation is caused by the finite duration of the photoemission process and results in "soft" edges; the density does not fall off to zero abruptly, but over a distance comparable with the laser pulse length [4]. The existence of waterbag bunches still needs to be proven experimentally. This requires a diagnostic method for determining whether an electron bunch is indeed a waterbag bunch, and to obtain a measure of the soft edges.

Coherent transition radiation (CTR), created by an electron bunch experiencing a sudden change in dielectric constant [7-9], is a well-known diagnostic tool to characterize the spatial distribution of electron bunches [10-13]. The bunch shape is always assumed to be Gaussian, in which case the CTR temporal electric field and power spectrum are known analytically [14,15]. This approach is mainly based on mathematical convenience, since generally there is little detailed knowledge of the actual bunch shape. The electric field spectrum of CTR radiated by a electron bunch with the shape of a sphere [16], and the form factor of a ellipsoidal electron bunch [17], are also known analytically but were never considered as a realistic option for diagnosing electron bunches. We extend the analytical theory of CTR to ellipsoidal electron bunches with soft edges, which include all bunch shapes from perfectly smooth Gaussians to hard-edged ellipsoids.
This makes the detection possible of realistic waterbag bunches. The calculations assume monoenergetic electron bunches with no angular spread.

The remainder of this paper is organized as follows. Section II starts with a short introduction of coherent transition radiation (CTR), emitted by electrons traveling perpendicularly through the interface between a perfect metal and vacuum. We introduce the form factor for any spatial distribution, in an analog fashion as in Refs. $[14,18,19]$. We only evaluate the coherent part of the spectrum and ignore the incoherent part, which is valid because of the large number of electrons $\left(\sim 10^{8}\right)$ present in a typical bunch. Section II ends with a discussion of the conditions under which the electric field spectrum of CTR, created at a dielectric-vacuum interface, can be described with the expressions for a perfect metal-vacuum interface.

With the general theory of CTR discussed, we focus on the specific situation of CTR generated by cylindrically symmetric ellipsoidal electron bunches traveling through a perfect metal-vacuum interface. In Sec. III we calculate the form factor of an ellipsoidal spatial distribution, both with soft and hard edges. In Sec. IV the temporal electric field of CTR is calculated. To make this calculation physically relevant, the frequency spectrum has to be cut off at high and low frequencies. The high frequency components of the coherent radiation are either cut off by the form factor or by the dielectric response of the medium. The low frequency components are cut off due to the finite extent of the dielectric, resulting in diffraction radiation [20]. To take this into account we use Babinet's [21] principle in Sec. IV to calculate the coherent diffraction radiation of a dielectric disk much larger than the transverse bunch size $[9,14]$. In Sec. V the influence of a finite energy spread and angular spread of the electron bunch on CTR is discussed. A summary is presented in Sec. VI.

\section{COHERENT TRANSITION RADIATION}

In this section a brief introduction of CTR is given. We assume that the electrons are traveling perpendicularly through an interface between a perfect metal and vacuum. 
At the end of this section we show that this is an accurate model for CTR in the $\mathrm{THz}$ regime, for any realistic metalvacuum interface.

\section{A. Electric fields of CTR}

As is well known [7], the electric field spectrum $E_{\theta}(\boldsymbol{r}, \omega)$ of transition radiation (TR) emitted into the vacuum, by a single electron traveling perpendicularly through a perfect metal-vacuum interface, is given by

$$
E_{\theta}(\boldsymbol{r}, \omega)=\frac{-e \beta \sin \theta}{(2 \pi)^{2} \varepsilon_{0} c\left(1-\beta^{2} \cos ^{2} \theta\right)} \frac{e^{i(\omega / c) r}}{r},
$$

where $e$ is the electronic charge, $c$ is the speed of light in vacuum, $\varepsilon_{0}$ is permittivity of free space, and $\beta=\frac{v}{c}$ with $v$ the velocity of the electron. The observation vector $r$ makes an angle $\theta$ with the $z$ axis. At $t=0$ the electron is located in the origin.

If the electron is located at $\boldsymbol{r}_{j}$ at $t=0$, we can write Eq. (1) for $\boldsymbol{r} \gg \boldsymbol{r}_{j}$ as

$$
\begin{aligned}
E_{\theta}(\boldsymbol{r}, \omega)= & \frac{-e \beta \sin \theta}{(2 \pi)^{2} \varepsilon_{0} c\left(1-\beta^{2} \cos ^{2} \theta\right)} \\
& \times \frac{e^{i(\omega / c) r}}{r} e^{-i(\omega / c) n \cdot r_{j}^{t}-i(\omega / v) z_{j}},
\end{aligned}
$$

with $\boldsymbol{n}=\frac{\boldsymbol{r}}{r}$ the unit vector in the direction of observation and $\boldsymbol{r}_{j}^{t}$ the projection of $\boldsymbol{r}_{j}$ on the $x-y$ plane. A sketch of the situation is shown in Fig. 1 for illustration.

Equation (2) can be used to calculate the CTR radiated by an bunch of $N$ electrons traveling through a perfect metal-vacuum interface. We define $\rho(\boldsymbol{r})$ as the electron density distribution of the bunch. We assume that all electrons travel in the $z$ direction with the same velocity, and that each electron is located at a different position $\boldsymbol{r}_{j}$ at $t=0$. The electric field spectrum of the total CTR can then

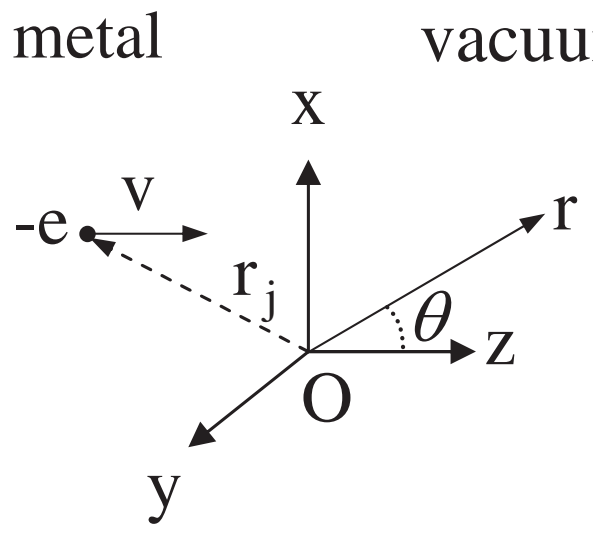

FIG. 1. Snapshot of electron traveling with speed $\boldsymbol{v}=\boldsymbol{v} \boldsymbol{e}_{z}$, taken at $t=0$ to illustrate the definition of the coordinates. The position of the electron at $t=0$ is $\boldsymbol{r}_{j}$. The half-space $z<$ 0 is occupied by a metal, the half-space $z>0$ is vacuum. The observation vector $\boldsymbol{r}$ makes an angle $\theta$ with the $z$ axis. be written as

$$
E_{\theta}(\boldsymbol{r}, \omega)=\frac{-e N \beta \sin \theta}{(2 \pi)^{2} \varepsilon_{0} c\left(1-\beta^{2} \cos ^{2} \theta\right)} f(\omega) \frac{e^{i(\omega / c) r}}{r} .
$$

The term $f(\omega)$ is called the form factor and is given by

$$
f(\omega)=\frac{1}{N} \sum_{j=1}^{N} e^{-i(\omega / c) \boldsymbol{n} \cdot \boldsymbol{r}_{j}^{t}-i(\omega / v) z_{j}}
$$

Because $N$ is typically very large, we can replace the summation in Eq. (4) with an integral over the electron distribution function $h(\boldsymbol{r}) \equiv \frac{\rho(\boldsymbol{r})}{-e N}$

$$
f(\omega)=\int h(\boldsymbol{r}) e^{-i(\omega / c) \boldsymbol{n} \cdot \boldsymbol{r}^{t}-i(\omega / v) z} d^{3} \boldsymbol{r} .
$$

Note that $h(\boldsymbol{r})$ is subject to the normalization condition

$$
\int h(\boldsymbol{r}) d^{3} \boldsymbol{r}=1 .
$$

Since we replaced the summation over the spatial distribution of the electron bunch with an integral over a wellbehaved continuous function, we cannot describe the incoherent part of the transition radiation with Eq. (5). Calculation of the incoherent part would require taking into account the exact position of each electron. However, the electron density distribution scales with $N$, while in general, the local density fluctuations scale with $\sqrt{N}$ [19]. Because of the large number of electrons in a typical bunch $\left(N \sim 10^{8}\right)$, the fluctuations and thus the incoherent radiation can safely be neglected. We will therefore proceed with the coherent radiation only.

\section{B. Power spectrum of CTR}

The total energy $W$ radiated into the vacuum can be calculated by integrating the Poynting vector over a surface in the far field, and over time

$$
W=\frac{1}{\mu_{0}} \iint \boldsymbol{E}(\boldsymbol{r}, t) \times \boldsymbol{B}(\boldsymbol{r}, t) \cdot d \boldsymbol{A} d t,
$$

where $d \boldsymbol{A}$ designates a surface area element in the far field and $\mu_{0}$ the permeability of free space. We can substitute the Fourier expansions of $\boldsymbol{E}(\boldsymbol{r}, t)$ and $\boldsymbol{B}(\boldsymbol{r}, t)$ into Eq. (7), and use the fact that only Fourier components of equal frequency give a contribution to the integration over time, resulting in the following expression:

$$
\frac{\partial^{2} W}{\partial \omega \partial \Omega}=4 \pi r^{2} \sqrt{\frac{\varepsilon_{0}}{\mu_{0}}}\left|E_{\theta}(\boldsymbol{r}, \omega)\right|^{2},
$$

for the energy radiated per unit frequency and unit solid angle into the vacuum.

\section{Perfect metal-vacuum interface}

It can be shown [8] that, as far as transition radiation is concerned, we can regard a dielectric as a perfectly con- 
ducting metal if the following condition is satisfied:

$$
\frac{1-\beta \cos \theta}{\sqrt{\varepsilon_{r}} \cos \theta} \ll 1,
$$

where $\varepsilon_{r}$ is the dielectric constant of the dielectric. Consequently, for all values of $\beta$ and $\varepsilon_{r}$, there always exists an angle $\theta$ at which the dielectric cannot be regarded as a metal. However, for $\gamma=\frac{1}{\sqrt{1-\beta^{2}}} \gg 1$ only a small fraction of the total radiation is emitted at those angles. Since the typical dielectric constant of a real metal is of the order of $10^{5}$ for frequencies in the THz regime [22], we can use the perfect metal-vacuum expression for CTR in the remainder of this paper, without seriously limiting the applicability of the theory.

\section{FORM FACTOR AND POWER SPECTRUM OF ELLIPSOIDAL ELECTRON BUNCHES}

In this section we evaluate the form factor for three different electron distributions with ellipsoidal symmetry. First, we evaluate the form factor of a uniformly filled ellipsoidal electron bunch with hard edges; second, for the case of an electron bunch with a Gaussian distribution; third, we use these two distributions to construct an electron distribution with ellipsoidal symmetry, that falls off smoothly from a uniform value to zero. This will serve as a model for a uniform filled ellipsoidal electron bunch with soft edges. To this purpose we write Eq. (5) as a onedimensional integral, by assuming that $h(\boldsymbol{r})$ is a function only of the radial coordinate of a spheroid; $R \equiv$ $\sqrt{x^{2}+y^{2}+\alpha^{2} z^{2}}$, where $\alpha$ is defined as the aspect ratio of the electron bunch in the lab frame. Note that the electron bunch can be oblate $(\alpha>1)$, spherical $(\alpha=1)$, and prolate $(0<\alpha<1)$. A spheroid is an ellipsoid having two equal semiaxes, in our case $x$ and $y$. We will refer to it as an ellipsoid in the remainder of this paper.

The form factor of an electron distribution with ellipsoidal symmetry can be written as

$$
f(\omega)=\frac{4 \pi}{\alpha} \int_{0}^{\infty} h(R) \sin \left(R \Theta \frac{\omega}{c}\right) \frac{R}{\Theta \frac{\omega}{c}} d R,
$$

where

$$
\Theta=(\alpha \beta)^{-1} \sqrt{\alpha^{2} \beta^{2} \sin ^{2} \theta+1} .
$$

The electron density distribution of a hard-edged uniformly filled ellipsoidal electron bunch is given by

$$
h_{e}(R)= \begin{cases}\frac{3 \alpha}{4 \pi R_{t}^{3}} & \text { if }|R| \leq R_{t} \\ 0 & \text { if }|R|>R_{t}\end{cases}
$$

where $R_{t}$ is the transverse radius of the ellipsoidal electron bunch. Substitution of Eq. (12) into Eq. (10) leads to the corresponding form factor [17]:

$$
f_{e}(\omega)=3 \frac{\sin \left(R_{t} \Theta \frac{\omega}{c}\right)-\left(R_{t} \Theta \frac{\omega}{c}\right) \cos \left(R_{t} \Theta \frac{\omega}{c}\right)}{\left(R_{t} \Theta \frac{\omega}{c}\right)^{3}} .
$$

The next is a Gaussian electron distribution,

$$
h_{g}(R)=\frac{\alpha}{(2 \pi)^{3 / 2} \sigma_{t}^{3}} e^{-\left[R^{2} /\left(2 \sigma_{t}^{2}\right)\right]}
$$

where $\sigma_{t}$ is the root-mean-square transverse dimension of the electron bunch. Substitution of Eq. (14) into Eq. (10) leads to the corresponding form factor

$$
f_{g}(\omega)=e^{-(1 / 2)\left[\sigma_{t} \Theta(\omega / c)\right]^{2}} .
$$

We can model a uniformly filled ellipsoidal electron bunch, that falls off smoothly from the uniform value to zero, by writing the electron distribution function as the convolution of a hard-edged distribution of size $R_{t}$ and a Gaussian distribution of size $\sigma_{t}$ :

$$
\begin{aligned}
h_{e \otimes g}(R) & =A h_{e} \otimes h_{g}=A \int_{-R_{t}}^{R_{t}} e^{-\left[(R-\xi)^{2} /\left(2 \sigma_{t}^{2}\right)\right]} d \xi \\
& =A \sqrt{\frac{\pi}{2}} \sigma_{t}\left[\operatorname{erf}\left(\frac{R+R_{t}}{\sqrt{2} \sigma_{t}}\right)-\operatorname{erf}\left(\frac{R-R_{t}}{\sqrt{2} \sigma_{t}}\right)\right],
\end{aligned}
$$

where erf is the error function [23]. The normalization constant is given by

$$
A=\frac{3 \alpha}{4 \sqrt{2} \pi^{3 / 2} R_{t}^{3} \sigma_{t}\left(1+3 \frac{\sigma_{t}^{2}}{R_{t}^{2}}\right.} .
$$

If we calculate the form factor by substituting Eq. (16) into Eq. (10), we obtain

$$
f_{e \otimes g}(\omega)=\frac{3}{\left(1+3 \frac{\sigma_{t}^{2}}{R_{t}^{2}}\right)} e^{-(1 / 2)\left[\sigma_{t} \Theta(\omega / c)\right]^{2}} \frac{\left[1+\left(\sigma_{t} \Theta \frac{\omega}{c}\right)^{2}\right] \sin \left(R_{t} \Theta \frac{\omega}{c}\right)-R_{t} \Theta \frac{\omega}{c} \cos \left(R_{t} \Theta \frac{\omega}{c}\right)}{\left(R_{t} \Theta \frac{\omega}{c}\right)^{3}}
$$

Note that Eq. (18) reduces to Eq. (13) in the limit of vanishing $\sigma_{t}$ :

$$
\lim _{\sigma_{t} \rightarrow 0} f_{e \otimes g}(\omega)=f_{e}(\omega)
$$

In the limit of vanishing $R_{t}$, Eq. (18) reduces to Eq. (15):

$$
\lim _{R_{t} \rightarrow 0} f_{e \otimes g}(\omega)=f_{g}(\omega) .
$$

The three different electron distributions and the corresponding form factors are plotted in Figs. 2(a) and 2(b) for $\sigma_{t}=0.2 R_{t}$, which has been chosen for illustrative purposes. If we compare $f_{e}$ with $f_{e \otimes g}$, we see that the latter is 

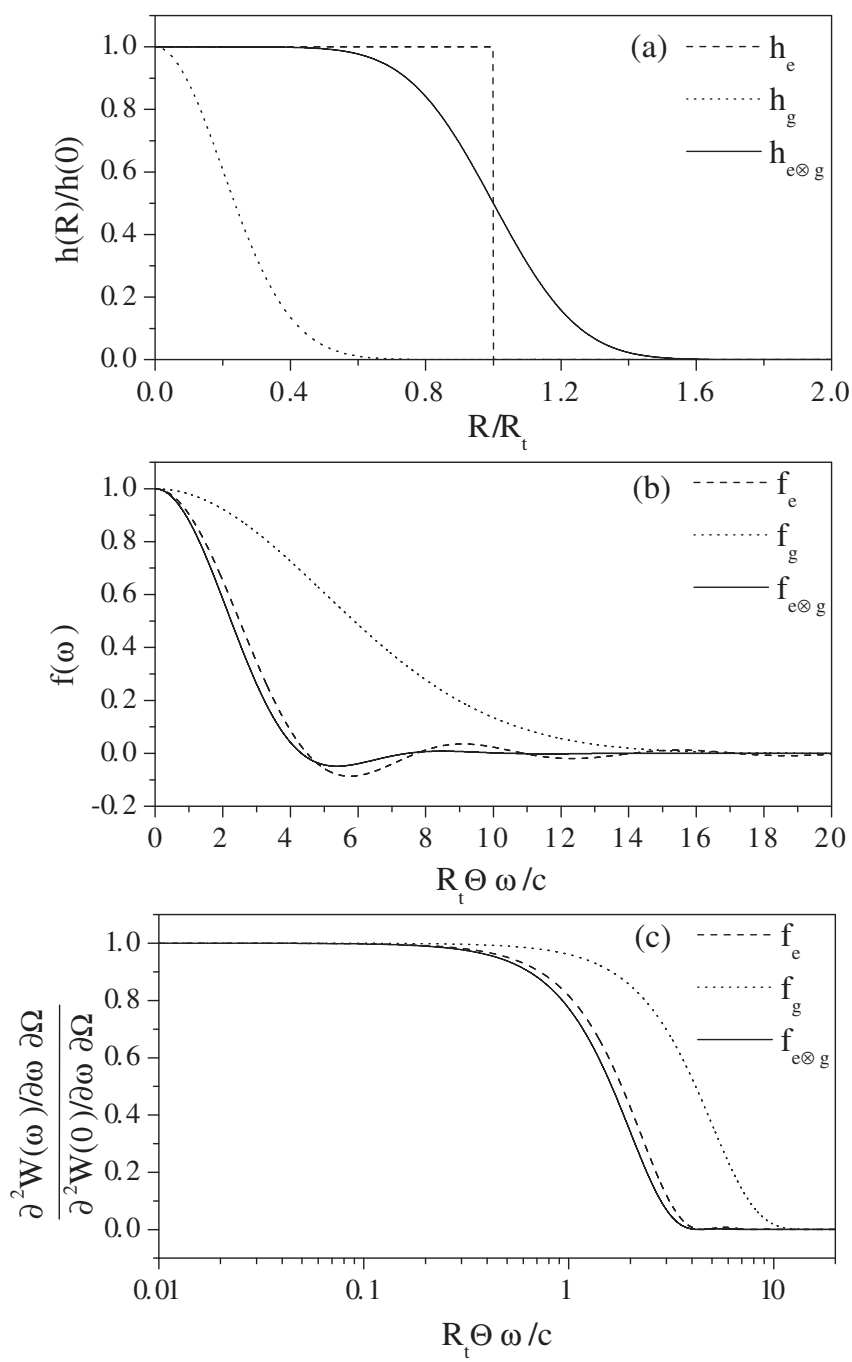

FIG. 2. Plot of the three different electron distributions (a), form factors (b), and power spectra (c) belonging to the different electron bunches discussed in this paper; the ellipsoidal electron bunch with hard edges $\left(h_{e}\right.$ and $\left.f_{e}\right)$, the Gaussian electron bunch $\left(h_{g}\right.$ and $f_{g}$ ), and the ellipsoidal electron bunch with soft edges $\left(h_{e \otimes g}\right.$ and $\left.f_{e \otimes g}\right)$. In all plots $\sigma_{t}=0.2 R_{t}$.

decreasing faster due to the soft edges. However, $f_{e \otimes g}$ is only slightly affected by the soft edges for frequencies $\omega \leq \frac{\sqrt{2} c}{\sigma_{t} \Theta}$.

The power spectrum can now easily be calculated using Eqs. (3), (8), (13), (15), and (18). Figure 2(c) shows a plot of the power spectrum, emitted by the three different electron density distributions.

\section{TEMPORAL ELECTRIC FIELD OF CTR GENERATED BY ELLIPSOIDAL ELECTRON BUNCHES}

To reconstruct the CTR pulse in space and time domain, the inverse Fourier transformation has to be performed:

$$
E_{\theta}^{h}(\boldsymbol{r}, t)=\int E_{\theta}^{h}(\boldsymbol{r}, \omega) e^{-i \omega t} d \omega
$$

If we substitute Eq. (3) into Eq. (21) we can write

$$
E_{\theta}^{h}(\boldsymbol{r}, t)=\frac{-e N \beta \sin \theta}{(2 \pi)^{2} \varepsilon_{0} c\left(1-\beta^{2} \cos ^{2} \theta\right)} \frac{1}{r} \int f(\omega) e^{-i \omega t_{0}} d \omega
$$

with $t_{0}=t-\frac{r}{c}$. At this point we need to address a problem with the form factor. The coherent power spectrum is nonzero at $\omega=0$ [see Fig. 2(c)]. According to Eq. (22) this implies a DC offset in the radiation pulse, which is physically impossible. This is a consequence of the assumption of an infinitely large metal-vacuum interface. In reality the polarization currents, responsible for the CTR, are spatially restricted to the transverse size of the metalvacuum interface. They therefore create coherent diffraction radiation (CDR) at the edges of the interface [20]. CDR will be generated only at wavelengths larger than the spatial dimensions of the interface, i.e., small $\omega$, since smaller wavelengths will add up incoherently. As we show below, CDR leads to the cancellation of CTR for $\omega \rightarrow 0$.

\section{A. Coherent diffraction radiation}

Diffraction radiation can be calculated by applying Kirchhoff's diffraction theory to the fields incident on the interface [21], in our case the fields of the electron bunch. For an electron traveling perpendicular through a circular aperture the expressions for diffraction radiation are well known [9], and can be used to model the finite extent of the perfect metal-vacuum interface, using Babinet's principle [21]. The necessary assumption of a circular interface is not a problem, we are only interested in the general influence of the CDR, independent of a particular geometry. Since a circular interface maintains the cylindrical symmetry of the problem, it is a natural choice. We choose the radius of the interface $a \gg R_{t}$. This allows us to model the bunch as a point particle with charge $-e N$, passing through the center of the circular interface. Under these assumptions we can write

$$
\begin{aligned}
E_{\theta}^{c r}(\boldsymbol{r}, \omega)= & \frac{-e N \beta \sin \theta}{(2 \pi)^{2} \varepsilon_{0} c\left(1-\beta^{2} \cos ^{2} \theta\right)} \frac{e^{i(\omega / c) r}}{r} \\
& \times f(\omega)[1-d(\omega)],
\end{aligned}
$$

for the spectrum of the electric field of the total coherent radiation $E_{\theta}^{c r}(\boldsymbol{r}, \omega)$, which describes both CTR and CDR. The function $d(\omega)$ describes the diffraction radiation and is given by $[9,14]$ 


$$
\begin{aligned}
d(\omega)= & J_{0}\left(a \sin \theta \frac{\omega}{c}\right)\left[\frac{a}{\beta \gamma} \frac{\omega}{c} K_{1}\left(\frac{a}{\beta \gamma} \frac{\omega}{c}\right)\right. \\
& \left.+\frac{1}{2}\left(\frac{a}{\beta \gamma} \frac{\omega}{c}\right)^{2} K_{0}\left(\frac{a}{\beta \gamma} \frac{\omega}{c}\right)\right] \\
& +\frac{1}{2}\left(\frac{a}{\beta \gamma} \frac{\omega}{c}\right)^{2} J_{2}\left(a \sin \theta \frac{\omega}{c}\right) K_{0}\left(\frac{a}{\beta \gamma} \frac{\omega}{c}\right),
\end{aligned}
$$

with $J_{m}$ and $K_{m}$ the $m$ th order regular and modified Bessel functions, respectively. Figure 3 shows a plot of the frequency dependence of $E_{\theta}^{c r}(\boldsymbol{r}, \omega)$ for a hard-edged ellipsoidal electron bunch. Note the break in the frequency scale, which serves to illustrate the spectrum at both low and high frequencies. The values in the upper right corner indicate the parameters used in the plot. The important feature of the plot, however, is the fact that $E_{\theta}^{c r}(\boldsymbol{r}, \omega) \rightarrow 0$, as $\omega \rightarrow 0$. The DC offset in the radiation is thus indeed removed by taking CDR into account.

The expression in Eq. (23) can be simplified to

$E_{\theta}^{c r}(\boldsymbol{r}, \omega)=\frac{-e N \beta \sin \theta}{(2 \pi)^{2} \varepsilon_{0} c\left(1-\beta^{2} \cos ^{2} \theta\right)} \frac{e^{i(\omega / c) r}}{r}[f(\omega)-d(\omega)]$.

This is allowed because $f(\omega) \simeq 1$ when $d(\omega) \neq 0$. This is a direct consequence of the assumption $R_{t} \ll a$, and is the mathematical consequence of treating the electron bunch as a pointlike particle with charge $-e N$, passing through the center of the circular interface. If we look at Eq. (25), we see that the total coherent radiation can be described by subtracting the CDR from the CTR, independent of the CTR. We can thus continue with calculating the temporal

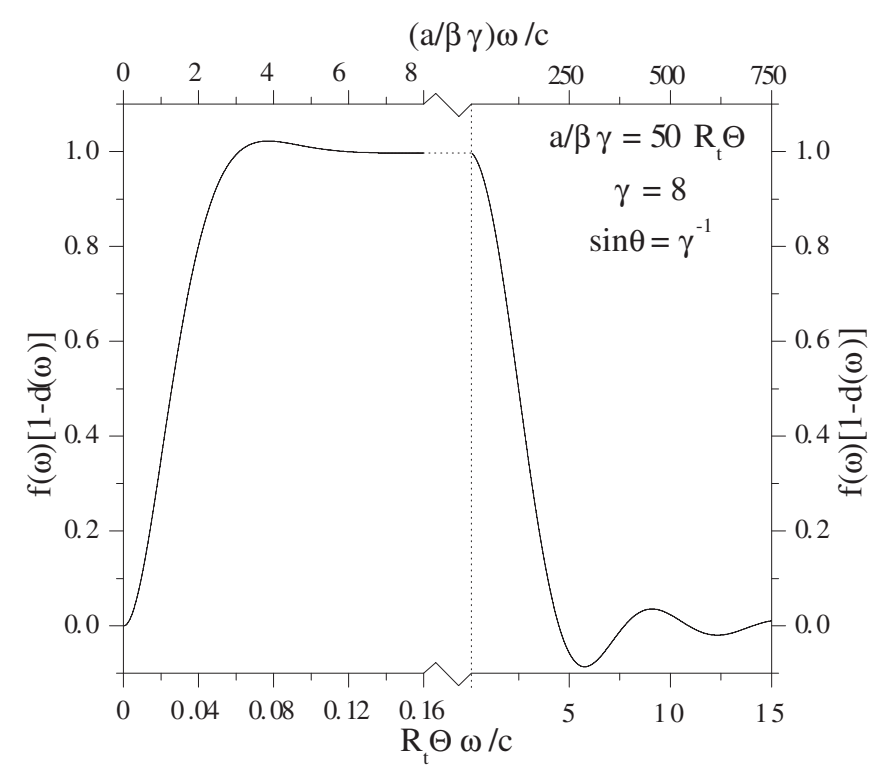

FIG. 3. Frequency dependence of the electric field spectrum of the total coherent radiation pulse due to a hard-edged ellipsoidal electron bunch, including both CTR and CDR. The parameters used to make the plot are listed in the upper right corner. electric field of CTR, by evaluating the inverse Fourier transform of $f(\omega)$, as long as we keep in mind that the CDR has to be subtracted. This means that we have to evaluate the inverse Fourier transform of $f(\omega)$ and $d(\omega)$.

\section{B. Inverse Fourier transform of $\boldsymbol{d}(\omega)$}

Evaluating the inverse Fourier transform of $d(\omega)$ analytically is not straightforward and outside the scope of this paper. Instead we will use the fast Fourier transform (FFT) algorithm to evaluate Eq. (23) numerically. This will illustrate the influence of CDR on CTR quite generally, as long as the assumption $R_{t} \ll a$ holds. Note that due to this assumption it makes no difference whether we calculate the FFT of Eq. (23) or Eq. (25); both produce the same result. The result of the FFT, for $f(\omega)=f_{e}(\omega)$, is shown in Fig. 4. The relevant parameters are listed in the upper left corner. We observe two half-cycle pulses superposed, with opposite polarity. The long negative pulse is CDR, the short positive pulse is CTR. The CTR pulse is shown in more detail in the upper right corner, its shape will be discussed later. The important feature of Fig. 4 is that the CDR pulse is almost constant on the time scale of the CTR pulse, and the CDR electric field strength is much lower. This conclusion holds generally as long as $\frac{a}{\beta \gamma} \gg R_{t} \Theta$. We will therefore proceed with the temporal CTR pulse only and neglect the CDR.

\section{Inverse Fourier transform of $\boldsymbol{f}(\boldsymbol{\omega})$}

Using Eqs. (15) and (18), the form factor of the ellipsoidal electron bunch with soft edges can be written as

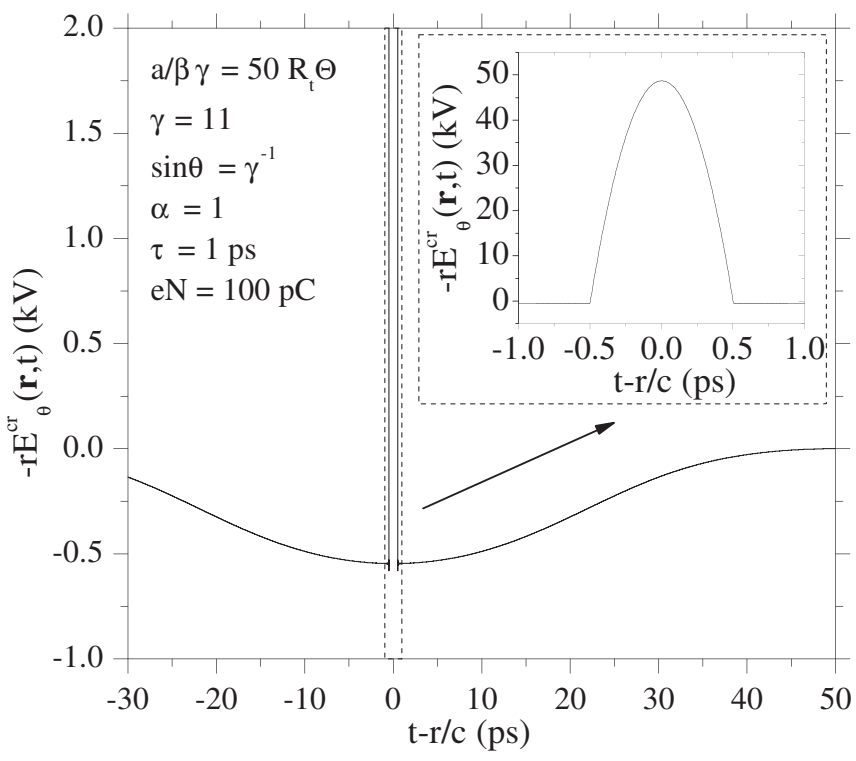

FIG. 4. Plot of temporal electric field, with coherent diffraction radiation taken into account. The long negative pulse is due to CDR. The CTR pulse is shown in the inset in the upper right corner. The parameters used to make the plot are listed in the upper left corner. 


$$
f_{e \otimes g}(\omega)=f_{e}^{\sigma_{t}}(\omega) f_{g}(\omega)
$$

where

$$
f_{e}^{\sigma_{t}}(\omega) \equiv \frac{3}{\left(1+3 \frac{\sigma_{t}^{2}}{R_{t}^{2}}\right)} \frac{\left[1+\left(\sigma_{t} \Theta \frac{\omega}{c}\right)^{2}\right] \sin \left(R_{t} \Theta \frac{\omega}{c}\right)-R_{t} \Theta \frac{\omega}{c} \cos \left(R_{t} \Theta \frac{\omega}{c}\right)}{\left(R_{t} \Theta \frac{\omega}{c}\right)^{3}}
$$

Since a product of Fourier components leads to a convolution in time domain, we can use Eqs. (26) and (27) to find the temporal electric field of the ellipsoidal electron bunch with soft edges.

However, we first calculate the temporal electric field of the ellipsoidal electron bunch with hard edges. We do this by calculating the inverse Fourier transform of $f_{e}^{\sigma_{t}}(\omega)$ and substituting $\sigma_{t}=0$ afterwards. The inverse Fourier transform of $f_{e}^{\sigma_{t}}$ is

$$
\begin{aligned}
& f_{e}^{\sigma_{t}}\left(t_{0}\right)=\int f_{e}(\omega) e^{-i \omega t_{0}} d \omega \\
& =\frac{\pi}{2} \frac{3}{1+3 \frac{\sigma_{t}^{2}}{R_{t}^{2}}} \frac{1}{\tau_{e}}\left(1+2 \frac{\sigma_{t}^{2}}{R_{t}^{2}}-\frac{t_{0}^{2}}{\tau_{e}^{2}}\right) \\
& \times \begin{cases}0 & \text { if }\left|t_{0}\right|>\tau_{e} \\
1 & \text { if }\left|t_{0}\right| \leq \tau_{e},\end{cases}
\end{aligned}
$$

with $\tau_{e}=\frac{R_{t} \Theta}{c}$ the duration of the CTR pulse, and $f_{e}^{\sigma_{t}}\left(t_{0}\right)$ the inverse Fourier transform of the form factor. If we assume $\sigma_{t}=0$ and substitute Eq. (28) into Eq. (22), we obtain $E_{\theta e}^{h}(\boldsymbol{r}, t)$, the CTR pulse of a hard-edged ellipsoidal electron bunch,

$$
\begin{gathered}
E_{\theta e}^{h}(\boldsymbol{r}, t)=\frac{-e N \beta \sin \theta}{(2 \pi)^{2} \varepsilon_{0} c\left(1-\beta^{2} \cos ^{2} \theta\right)} \times \\
\frac{3 \pi}{2 \tau_{e} r}\left(1-\frac{t_{0}^{2}}{\tau_{e}^{2}}\right) \times \begin{cases}0 & \text { if }\left|t_{0}\right|>\tau_{e} \\
1 & \text { if }\left|t_{0}\right| \leq \tau_{e} .\end{cases}
\end{gathered}
$$

Note that $E_{\theta e}^{h}(\boldsymbol{r}, t)$ has a parabolic profile with a length of $\tau_{e}$, which corresponds with the CTR pulse calculated with the FFT shown in Fig. 4.

Next we calculate the inverse Fourier transform of $f_{g}(\omega)$,

$$
f_{g}\left(t_{0}\right)=\int f_{g}(\omega) e^{-i \omega t_{0}} d \omega=\frac{\sqrt{2} \bar{\pi}}{\tau_{g}} e^{\left.-(1 / 2)\left[t_{0} / \tau_{g}\right)^{2}\right]},
$$

with $\tau_{g}=\frac{\sigma_{t} \Theta}{c}$. The CRT pulse in the case of a Gaussian electron bunch $E_{\theta g}^{h}(\boldsymbol{r}, t)$ is thus [20]

$$
E_{\theta g}^{h}(\boldsymbol{r}, t)=\frac{-e N \beta \sin \theta}{(2 \pi)^{2} \varepsilon_{0} c\left(1-\beta^{2} \cos ^{2} \theta\right)} \frac{\sqrt{2 \pi}}{\tau_{g} r} e^{-(1 / 2)\left[\left(t_{0} / \tau_{g}\right)^{2}\right]} .
$$

We can now find $E_{\theta e \otimes g}^{h}(\boldsymbol{r}, t)$, the temporal electric field of the electron bunch with soft edges. To do so we write

$$
f_{e \otimes g}\left(t_{0}\right)=\frac{1}{2 \pi} f_{e}^{\sigma_{t}}\left(t_{0}\right) \otimes f_{g}\left(t_{0}\right)
$$

which leads to

$$
E_{\theta e \otimes g}^{h}(\boldsymbol{r}, t)=\frac{-e N \beta \sin \theta}{(2 \pi)^{2} \varepsilon_{0} c\left(1-\beta^{2} \cos ^{2} \theta\right)} \frac{1}{r} \frac{1}{2 \pi} f_{e}\left(t_{0}\right) \otimes f_{g}\left(t_{0}\right) .
$$

The result of the convolution in Eq. (33) is

$$
\begin{aligned}
\frac{1}{2 \pi} f_{e}\left(t_{0}\right) \otimes f_{g}\left(t_{0}\right)= & \frac{1}{2 \pi} \int_{-\infty}^{\infty} f_{e}(\tau) f_{g}\left(t_{0}-\tau\right) d \tau \\
= & \sqrt{\frac{\pi}{8}} \frac{3}{1+3 \frac{\tau_{g}^{2}}{\tau_{e}^{2}}} \frac{1}{\tau_{e}}\left\{\frac{\tau_{g}}{\tau_{e}} e^{-\left[\left(t_{0}+\tau_{e}\right)^{2} /\left(2 \tau_{g}^{2}\right)\right]}\left[1-\frac{t_{0}}{\tau_{e}}+\left(1+\frac{t_{0}}{\tau_{e}}\right) e^{2\left[\left(t_{0} \tau_{e}\right) / \tau_{g}^{2}\right]}\right]\right. \\
& \left.+\sqrt{\frac{\pi}{2}}\left(\frac{\tau_{g}^{2}}{\tau_{e}^{2}}+1-\frac{t_{0}^{2}}{\tau_{e}^{2}}\right)\left[\operatorname{erf}\left(\frac{t_{0}+\tau_{e}}{\sqrt{2} \tau_{g}}\right)-\operatorname{erf}\left(\frac{t_{0}-\tau_{e}}{\sqrt{2} \tau_{g}}\right)\right]\right\}
\end{aligned}
$$

Figure 5 shows a plot of CTR pulses, corresponding to a Gaussian, a hard-edged, and a soft-edged ellipsoidal electron density distribution. The contribution of CDR is not taken into account in the figure but can simply be added, as discussed in Sec. IV B. We observe three half-cycle pulses. We furthermore observe that $E_{\theta g}^{h}(\boldsymbol{r}, t)$ has the highest peak electric field, because it is the shortest bunch. If we look at $E_{\theta e \otimes g}^{h}(\boldsymbol{r}, t)$, we see that the soft edges in the electron bunch lead to soft edges in the temporal profile of the CTR pulse. The peak electric field is less with respect to $E_{\theta e}^{h}(\boldsymbol{r}, t)$, which is a consequence of the larger spatial dimension of the electron bunch due to the soft edges. The shape of all three CTR pulses appears to be a direct fingerprint of the corresponding electron density distribution. Note, however, that the CTR pulse length depends on $\theta$ [see Eq. (11)]. 


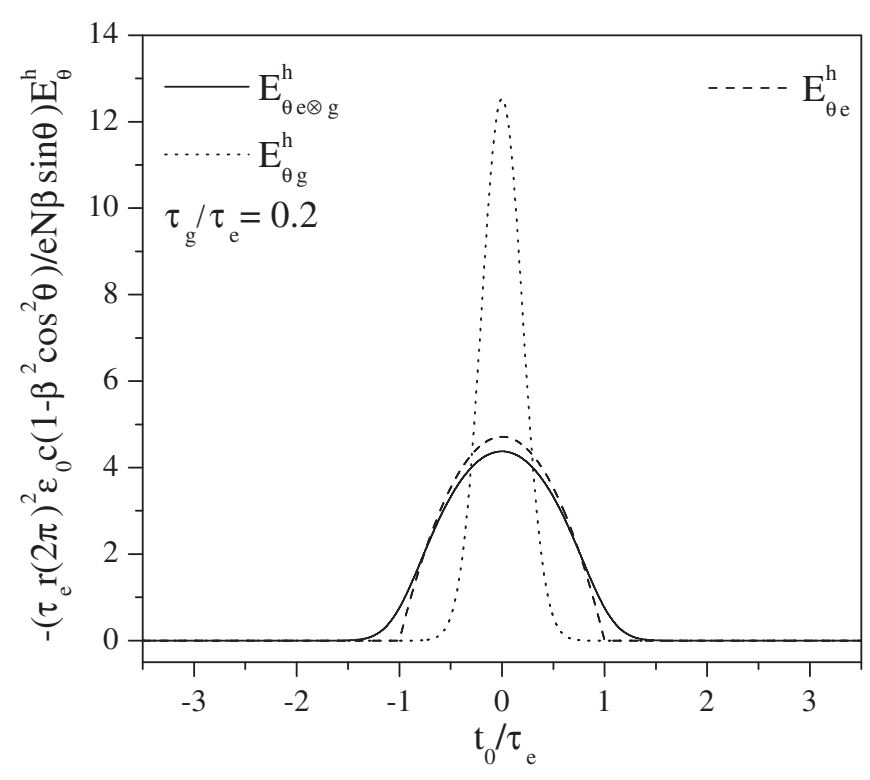

FIG. 5. Plot of the temporal electric field of the CTR pulse, for the three different electron bunches discussed in this article; the ellipsoidal electron bunch with hard edges $E_{\theta e}^{h}$, the Gaussian electron bunch $E_{\theta g}^{h}$, and the ellipsoidal electron bunch with soft edges $E_{\theta e \otimes g}^{h}$. The choice $\tau_{g}=0.2 \tau_{e}$ has been made for illustrative purposes.

\section{INFLUENCE OF ENERGY AND ANGULAR SPREAD OF ELECTRON BUNCH}

The calculations of CTR in this article assumed monoenergetic electron bunches with no angular spread. Here the conditions are discussed, under which this is valid.

A finite angular spread can affect the CTR pulse in two ways: it can lead to decoherence, and thus less power; and it can change the angular distribution of the radiation pulse. The phase differences are induced because the electrons pass the interface at different times and different transverse coordinates. Clearly, if the angular spread $\Delta \vartheta$ of the electron bunch is much smaller than the aspect ratio $\alpha$ of the bunch, $\Delta \vartheta \ll \alpha$, then decoherence due to a finite angular spread can be neglected. Since TR is emitted at angles of $\sim \gamma^{-1}$, the angular distribution is not affected if $\Delta \vartheta \ll \gamma^{-1}$.

A finite energy spread has a similar effect on the CTR pulse as a finite angular spread. The influence on the decoherence, caused by the different time of passage of the electrons, can be neglected if the relative velocity spread $\frac{\Delta v}{v}=\frac{1}{\gamma^{2}-1} \frac{\Delta \gamma}{\gamma} \ll 1$. The angular distribution is not affected by the energy spread if $\frac{\Delta \gamma}{\gamma} \ll 1$, because TR is emitted at angles of $\sim \gamma^{-1}$.

\section{SUMMARY}

We have analytically calculated the electric field spectrum, power spectrum, and temporal electric field of CTR, created by ellipsoidal electron bunches, both with hard and soft edges.

The theory described in this paper can be used for diagnosing ellipsoidal electron bunches. These bunches are of major interest because of their linear self-fields, which would solve the problem of space-charge induced emittance degradation.

[1] O. D. Kellogg, Foundations of Potential Theory (SpringerVerlag, Berlin, 1926).

[2] I. M. Kapchinskii and V.V. Vladimirskii, in Proceedings of the International Conference on High Energy Accelerators (CERN, Geneva, 1959), p. 247.

[3] L. Serafini, in Towards X-Ray Free Electron Lasers: Workshop on Single Pass, High Gain FELs Starting from Noise, Aiming at Coherent X-Rays, edited by R. Bonifacio and W. A. Barletta, AIP Conf. Proc. No. 413 (AIP, New York, 1997), p. 321.

[4] O.J. Luiten, S.B. van der Geer, M.J. de Loos, F. B. Kiewiet, and M. J. van der Wiel, Phys. Rev. Lett. 93, 094802 (2004).

[5] O.J. Luiten, S.B. van der Geer, M.J. de Loos, F. B. Kiewiet, and M.J. van der Wiel, in Proceedings of the European Particle Accelerator Conference, Lucerne (EPS-AG, Lucerne, 2004), p. 725.

[6] S. B. van der Geer, M.J. de Loos, T. van Oudheusden, W.P.E.M. Op 't Root, M.J. van der Wiel, and O.J. Luiten, Phys. Rev. ST Accel. Beams 9, 044203 (2006).

[7] V.L. Ginzburg and I.M. Frank, Sov. Phys. JETP 16, 15 (1946).

[8] V. Ginzburg and V. Tsytovich, Transition Radiation and Transition Scattering (Hilger, London, 1990).

[9] M. Ter-Mikaelian, High-Energy Electromagnetic Processes in Condensed Media (Wiley-Intersience, New York, 1972).

[10] U. Happek, A. J. Sievers, and E. B. Blum, Phys. Rev. Lett. 67, 2962 (1991).

[11] Y. Shibata, T. Takahashi, T. Kanai, K. Ishi, M. Ikezawa, J. Ohkuma, S. Okuda, and T. Okada, Phys. Rev. E 50, 1479 (1994).

[12] P. Kung, H.-C. Lihn, H. Wiedemann, and D. Bocek, Phys. Rev. Lett. 73, 967 (1994).

[13] A. Tremaine, J. B. Rosenzweig, S. Anderson, P. Frigola, M. Hogan, A. Murokh, C. Pellegrini, D. C. Nguyen, and R. L. Sheffield, Phys. Rev. Lett. 81, 5816 (1998).

[14] C. B. Schroeder, E. Esarey, J. van Tilborg, and W.P. Leemans, Phys. Rev. E 69, 016501 (2004).

[15] J. van Tilborg, C. B. Schroeder, C. V. Filip, C. Tóth, C. G. R. Geddes, G. Fubiani, R. Huber, R. A. Kaindl, E. Esarey, and W.P. Leemans, Phys. Rev. Lett. 96, 014801 (2006).

[16] B. M. Bolotovskii and A.V. Serov, Tech. Phys. 47, 1 (2002).

[17] Y. O. Averkov and V. M. Yakovenko, Plasma Phys. Rep. 30, 519 (2004).

[18] J. S. Nodvick and D. S. Saxon, Phys. Rev. 96, 180 (1954). 
[19] C. J. Hirschmugl, M. Sagurton, and G. P. Williams, Phys. Rev. A 44, 1316 (1991).

[20] J. van Tilborg, C. B. Schroeder, E. Esarey, and W. P. Leemans, Laser Part. Beams 22, 415 (2004).

[21] J.D. Jackson, Classical Electrodynamics (Wiley, New York, 1999).
[22] N. W. Ashcroft and N.D. Mermin, Solid State Physics (Brooks/Cole Thomson Learning, Belmont, MA, 1976).

[23] M. Abramowitz and I. A. Stegun, Handbook of Mathematical Functions (Dover Publications, Inc., New York, 1965). 